\title{
I sistemi informativi per il controllo e la valutazione delle performance nelle università italiane
}

\author{
Antonella Paolini* e Michela Soverchia*
}

\begin{abstract}
Information systems for control and performance evaluation in Italian universities

Law no. 240/2010 and subsequent legislation imposed on Italian state universities relevant changes to their governance and control systems. This phenomenon has created debates, studies and research on the consequent effects of changes, still going. Furthermore, universities are, especially in Italy, public administrations interesting and peculiar, characterized by common elements to other public entities, but also by distinctive and almost unique features.

This issue of Management Control includes six papers related the performance measurement and evaluation in the Italian universities. This issue is analyzed for as performance should be according to recent legislation, but also for how it should be according to the management and accounting principles not adopted by law, which could support internal decision-making processes (at the single university level) and external (at ministerial level, in terms of public policies).

Some critical issues emerged: the difficult integration of information systems useful for control and decisions; the habit to use cash accounting, with no consideration of operations' economic aspects, it's hard to overcome as a mentality and organizational processes; past practices in measuring/assessing teaching and research processes determined their excessive autonomy, while they should be integrated with each other and with the overall universities' functioning.
\end{abstract}

Keywords: Performance Measurement Systems, Italian Universities, Performance plans, disclosure, accrual accounting, performance-based funding, social reports.

* Dipartimento di Economia e Diritto, Università degli Studi di Macerata, antonella.paolini@unimc.it; michela.soverchia@unimc.it.

Management Control (ISSN 2239-0391, ISSNe 2239-4397), 2017, 1

DOI: 10.3280/MACO2017-001001 


\section{Il cambiamento dei sistemi di governo e controllo delle università}

La Legge n. 240/2010 e i successivi decreti attuativi hanno imposto alle università statali italiane rilevanti cambiamenti dei loro assetti di governo e di controllo. Il fenomeno ha dato vita ad una stagione di dibattiti, studi e ricerche sugli effetti dei cambiamenti verificatisi ed ancora in corso. Peraltro le università sono, specie in Italia, amministrazioni pubbliche (AP) interessanti e peculiari, contraddistinte da elementi comuni ad altre AP, ma anche da tratti assolutamente peculiari e per certi versi unici.

Il presente numero di Management Control accoglie una prima parte dei molteplici articoli sulla gestione ed il controllo nelle università italiane pervenuti alla Rivista. Vista la copiosità dei lavori pervenuti, l'editore tornerà nei prossimi numeri della Rivista su tali tematiche.

I sei articoli sono legati, a parere di chi li ha collazionati, dalla "misurazione e valutazione delle performance" diversamente declinate ma comunque presenti nelle università italiane.

\section{La misurazione e valutazione delle performance: criticità di gestione interna, comunicazione e interpretazione esterna}

Il primo articolo di Natalia Aversano, Francesca Manes Rossi, Paolo Tartaglia Polcini, I sistemi di misurazione della performance nelle università: considerazioni critiche sul sistema italiano, è quello che tratta la tematica più ampia e generale. Esso offre una panoramica dei sistemi di misurazione della performance adottati nella maggior parte dei paesi europei, soffermandosi - con un'analisi critica - sul sistema italiano, con particolare riferimento alle linee guida di recente emanate dall'Agenzia Nazionale per la Valutazione del Sistema Universitario e della Ricerca (ANVUR) in relazione all'attività amministrativa.

Un sistema pervaso da un processo di cambiamento che conduce le università verso una progressiva autonomia sul piano organizzativo e amministrativo (da un modello burocratico ad un modello manageriale) ma con ancora forte dipendenza sul piano delle risorse finanziarie. I sistemi informativi a sostegno di tale processo sono altresì sostenuti da precisi riferimenti normativi, ad esempio è stato modificato il sistema contabile, segnando il passaggio da un sistema di contabilità finanziaria (COFIN) ad uno economico-patrimoniale (COEP) (Paolini e Soverchia, 2013; Mussari e Sostero, 2014; Mussari et al., 2015; Trequattrini et al., 2015). Non sembra, però, che i sistemi di misurazione delle performance abbiano beneficiato fino in 
fondo della "produzione di alcuni dati": il controllo-guida (Paolini, 2003; Cantele et al., 2012) ancora manca e quindi le strategie, i programmi e le attività ancora non utilizzano i dati trasformati in informazioni (Marchi e Greco, 2012). Sicuramente ci sarà bisogno di "rodare", assestare e, soprattutto, integrare detti sistemi; un'integrazione che manca e gli autori ritengono che la causa possa essere individuata nella peculiarità delle università, dove si continuano a tenere separati i profili della didattica e della ricerca rispetto all'attività amministrativa anche in fase di misurazione e valutazione degli output erogati. In aggiunta, si debbono valutare le performance delle strutture organizzative (tipicamente i dipartimenti) all'interno delle quali coesistono, verso il conseguimento di obiettivi comuni, il personale docente e non docente.

Il secondo articolo di Alessandra Allini, Adele Caldarelli, Rosanna Spanò, La disclosure nei Piani delle Performance delle Università italiane. Intenti simbolici vs approcci integrati alla gestione, analizza, come il precedente, i Piani delle Performance, ma focalizza l'attenzione sulla loro capacità di comunicare contenuti importanti e sostanziali secondo regole di trasparenza e, di nuovo, verso approcci integrati con altri strumenti di programmazione e controllo.

Il Piano delle Performance, al di là di una sintetica menzione normativa e del modello proposto dalla Commissione Indipendente per la Valutazione, l'Integrità e la Trasparenza (CIVIT), è essenzialmente di natura discrezionale, consentendo margini di manovra agli organi di governo dell'università. Quali possono essere i contenuti informativi per comprendere se la comunicazione risulti sostanziale e, dunque, se le informazioni veicolate nei documenti siano idonee a dar corso a finalità di crescente trasparenza e di maggiore responsabilità amministrativa?

Le autrici cercano di rispondere con un'analisi empirica: lo studio di 66 Piani della Performance di atenei italiani conducono a risultati interessanti.

I Piani delle Performance, chiaramente con alcune eccezioni, mostrano: una scarsa valenza programmatica, ovvero un limitato coordinamento con il ciclo della programmazione e di bilancio e una mancanza d'informazioni su aree essenziali dell'attività strategica pianificata. In particolare, i contenuti sono poveri di questioni relative alle azioni di monitoraggio, ai temi della trasparenza e della prevenzione della corruzione. L'atteggiamento medio è di una compliance formale rispetto alla norma.

In chiave propositiva le autrici si aspettano un miglioramento, tanto che nel Piano delle Performance dovrebbero essere evidenti gli utilizzi di "strumenti formalizzati della pianificazione strategica, segnalatori di un ap- 
proccio integrato alla gestione, in grado di riflettere la vision dell'ateneo, condivisa a tutti i livelli organizzativi e, ancor più, da condividere con gli stakeholders in ragione del principio di trasparenza e accountability". Miglioramenti che potrebbero essere minati da una pressione normativa che, negli ultimi anni, è progressivamente cresciuta, tanto da innescare prassi di risposta poco adeguate (ai limiti della superficialità) o basate sull'insofferenza di dare una risposta alle richieste di legge.

Il terzo articolo di Sabrina Gigli e Marco Tieghi, La riforma del sistema informativo-contabile degli atenei Italiani. Una prima analisi delle situazioni aziendali emergenti dal nuovo modello di bilancio, approfondisce gli aspetti del bilancio d'esercizio delle università e li utilizza per comprendere le performance economico-finanziarie.

Il passaggio dalla COFIN alla COEP segna un cambiamento radicale che dovrebbe portare a rivedere i processi organizzativi e decisionali interni e ad offrire informazioni più fruibili agli stakeholders esterni.

Gli autori propongono: schemi di riclassificazione per lo stato patrimoniale e per il conto economico e un sistema di indici adattati alle specificità degli atenei; una rielaborazione dei bilanci ufficiali pubblicati sui siti web delle singole università censite; un'analisi per cogliere le differenze sostanziali derivanti dalle diverse modalità di contabilizzazione. Infatti, nonostante le disposizioni di legge inerenti schemi e principi contabili, gli autori osservano che esiste una rilevante eterogeneità dei comportamenti contabili assunti in seno ai vari atenei, un insuccesso della riforma contabile volta a perseguire omogeneità - dunque confrontabilità - dei dati presenti nei bilanci d'esercizio. Molteplici sono le cause illustrate dagli autori: tecnicocontabili, informative/informatiche, di ritardo normativo, organizzative, non ultimo di cultura economico-aziendale.

Dalle prime analisi emerge dunque una scarsa assimilazione delle logiche di funzionamento del nuovo sistema contabile all'effettivo impiego delle opportunità che esso offre a supporto della gestione, estensivamente intesa fino alla pianificazione e programmazione.

Lo stato di salute economico-finanziaria degli atenei sui cui bilanci sono state effettuate le elaborazioni (19 università che nel 2015 avevano collocato sul loro sito web l'intero "pacchetto" di bilancio d'esercizio) potrebbe dirsi nel complesso soddisfacente, seppure con alcune eccezioni.

Certe evidenze potrebbero essere di supporto in sede di una possibile revisione del quadro normativo adottato, con particolare riferimento a talune disposizioni in tema di: criteri di valutazione, ammortamento e rappresentazione delle poste di bilancio. Gli autori propongono: maggiore chia- 
rezza dei meccanismi di valorizzazione degli investimenti già in essere; possibilità di utilizzo di riserve e fondi iscritti nel patrimonio netto; definizione di univalenti coefficienti di ammortamento per le diverse tipologie di immobilizzazioni da assumere come "ordinario riferimento"; una maggiore chiarezza o assertività sul trattamento contabile e sulle modalità di rappresentazione in bilancio di talune poste; obbligo di un formato specifico per la predisposizione della Nota integrativa e della Relazione sulla gestione.

Il quarto articolo di Isabella Mozzoni, Simone Fanelli, Antonello Zangrandi, Il confronto di performance economico-finanziarie nelle università: un possibile modello di analisi per indici, si focalizza anch'esso sul "nuovo" bilancio d'esercizio degli atenei, allo scopo di approfondirne l'analisi economico-finanziaria. Molte sono le considerazioni concordanti con l'articolo precedente cui gli autori giungono dopo l'analisi empirica: parziale confrontabilità dei dati derivanti dai bilanci di esercizio (dalla lettura dei bilanci dei 16 atenei che erano in COEP nel 2014, emergono comportamenti molto eterogenei riguardo numerose poste); necessità di disporre di formati standardizzati per le parti meno quantitative del pacchetto informativo.

Gli autori insistono molto sulla necessità di definire un modello di analisi delle performance economico-finanziarie degli atenei in un'ottica di benchmark. Di fatto lo propongono e lo testano su 5 università anticipatrici dell'applicazione dei nuovi strumenti contabili. Il suddetto modello è tale che dovrebbe anche consentire il miglioramento dei sistemi decisionali che si avvalgono delle misure di performance economiche e finanziarie, compiendo quindi una riflessione non solo sulla qualità delle attività svolte, ma anche della sostenibilità economico-finanziaria dell'ente.

In una logica di controllo a sostegno delle informazioni utili per le decisioni, il modello potrebbe essere un punto di partenza per ragionare sull'utilizzo di strumenti gestionali consolidati ed efficaci sia per la governance dei singoli atenei, sia per i policy makers nella valutazione complessiva del sistema. I tradizionali sistemi di controllo, basati - anche nelle università statali - sul rispetto formale delle regole e degli adempimenti, non sono più sufficienti, ma occorrono strumenti di controllo in grado di fornire un giudizio sintetico sul raggiungimento delle condizioni di economicità dell'università. In tal senso l'analisi per indici di bilancio dovrebbe consentire all'ateneo di valutare il raggiungimento delle condizioni di equilibrio economico-finanziario nel medio-lungo periodo. In chiave strategica, la stessa analisi dovrebbe connotarsi nella dimensione "spaziale", cioè nel raffronto con le università aventi caratteristiche simili, o nel raffronto con in- 
dicatori medi. Il benchmarking è una vitale attività manageriale per supportare i decision maker degli atenei nel prendere decisioni strategiche.

Il percorso, come premesso, non è semplice, ma agli autori si sono impegnati su alcuni fronti: hanno considerato le peculiarità nella gestione e organizzazione degli atenei per meglio qualificare costi, oneri, ricavi e proventi in relazione ai processi gestionali che li generano. Si sono altresì imbattuti sul difforme utilizzo della COEP auspicando suggerimenti normativi per un'uniforme rilevazione contabile che consenta una comparabile lettura del bilancio d'esercizio. L'uniforme e corretta soluzione sarebbe di vantaggio per: rendere più responsabili i dirigenti sui risultati conseguiti; controllare i costi per migliorare i livelli di efficienza e monitorare la spesa pubblica; ottenere una rappresentazione affidabile del patrimonio pubblico, così da consentire una gestione più accurata e razionale (Agasisti e Catalano 2013; Paolini e Soverchia 2013; Mussari e Sostero, 2014).

\section{La misurazione e valutazione delle performance: allocazione interna delle risorse e rendicontazione sociale}

Il quinto articolo di Andrea Francesconi ed Enrico Guarini, Perfomance-based funding e sistemi di allocazione delle risorse ai dipartimenti: prime evidenze nelle università italiane, sposta l'attenzione sulle logiche di assegnazione delle risorse nelle principali strutture organizzative delle università, vale a dire i dipartimenti.

La cornice teorica di riferimento è quella dei sistemi di misurazione e valutazione delle performance (PMS) nell'ambito delle AP, sistemi volti a migliorare le performance delle organizzazioni (Lawson et al., 2003, De Bruijn, 2002). Molte amministrazioni hanno quindi, nel tempo, introdotto PMS nella convinzione che questi avrebbero portato a miglioramenti nei risultati ottenuti e ad una più efficace accountability interna ed esterna dell'organizzazione. Un ulteriore riferimento teorico è quello dall'emulazione delle logiche di mercato nell'ambito pubblico tramite l'introduzione di meccanismi di performance-based funding (PBF), ovvero il finanziamento delle aziende basato sulle performance, tema che ricade nell'ambito del New Public Management. Il PBF è da annoverare tra gli strumenti più utilizzati dai governi per il finanziamento delle università. Ciò, a partire dal 2014, è avvenuto anche nell'ambito del sistema universitario italiano; esso è, per il momento, un meccanismo parziale, ma destinato a sostituire progressivamente il finanziamento basato sulla spesa storica. 
Gli autori hanno realizzato un'indagine empirica sui meccanismi interni di allocazione di risorse particolari: i punti organico (PO) per le assunzioni e/o passaggi di carriera nei dipartimenti. È possibile, infatti, identificare uno stretto collegamento tra le dimensioni di performance dell'ateneo considerate nel PBF, la relativa assegnazione su base competitiva dei PO e il contributo effettivo dei dipartimenti alla performance complessiva.

I sistemi di allocazione delle risorse basati sulla performance sono, secondo gli autori, ampiamente utilizzati dagli atenei italiani e la loro progettazione è influenzata dal PBF nazionale e dai relativi indicatori. Ciò nonostante, l'influenza del PBF sembra essere solo parziale, in quanto la rilevanza ed il peso degli indicatori di performance utilizzati dagli atenei analizzati non sono strettamente legati a quelli impiegati nel sistema nazionale.

I sistemi analizzati presentano le seguenti caratteristiche: sono multidimensionali (diverse le misurazioni di performance utilizzate) e bilanciati; sono focalizzati nell'indirizzare i comportamenti del dipartimento e del corpo accademico verso il perseguimento degli obiettivi strategici dell'ateneo; sono coerenti con l'assetto organizzativo esistente.

Il lavoro, che si è avvalso di interviste ai decision maker delle università selezionate per l'analisi empirica, consente di evidenziare un elevato grado di consapevolezza sui possibili effetti distorsivi di una meccanicistica applicazione degli indicatori nazionali nell'ambito dei processi di allocazione delle risorse ai dipartimenti.

Sono invece differenti i sistemi di allocazione delle risorse in termini di focus, misure impiegate e formule utilizzate. Le università di dimensioni inferiori si caratterizzano per un utilizzo più limitato di algoritmi e formule, ritenendo altri strumenti di management più efficaci nel garantire l'efficacia e la qualità dei processi decisionali; gli atenei di dimensioni maggiori attribuiscono ai PMS un maggior peso al fine di limitare la discrezionalità dei processi decisionali e garantire agli stessi un adeguato livello di trasparenza.

Gli autori non sono riusciti a comprendere come i PMS in uso possano spiegare le diverse performance degli atenei italiani. Ad esempio, buone performance delle attività di ricerca possono essere ottenute anche in assenza di PMS formalizzati di tipo quantitativo, sia negli atenei di dimensioni maggiori, sia negli atenei più piccoli. È emerso come attribuire un particolare rilievo alla Valutazione della Qualità della Ricerca (VQR) nei parametri di PMS non ne determini necessariamente ed automaticamente un miglioramento; piuttosto, sembra che questo si colleghi più ad altri elementi e valori, quali la qualità, l'etica, la dedizione del corpo docente al proprio 
lavoro, ecc.). Analogamente, gli stessi PMS analizzati non spiegano la complessità e specificità dei dipartimenti e delle relative aree disciplinari.

Il sesto ed ultimo articolo di Federica Farneti, Benedetta Siboni, Carlotta Del Sordo, La misurazione della performance sociale nell'esperienza dell'Università di Bologna, sposta l'attenzione sulla rendicontazione sociale che, nell'ultimo decennio, ha interessato anche il settore universitario. Le autrici osservano che nel 2009, in Italia, 12 università realizzavano un bilancio sociale come strumento volontario (Del Sordo, Siboni, 2013), ma ad oggi il numero è quasi raddoppiato. Contestualmente riscontrano una mancanza di ricerche empiriche sul tema e cercano di sopperire con un case study, quello dell'Università di Bologna, un ateneo di grandi dimensioni che può contare su una storia di rendicontazione estesa, tale da condurre alla definizione di un bilancio sociale completo nelle sue finalità informative esterne ed interne.

È stato strutturato il percorso di rendicontazione sociale partendo dall'iter triennale di produzione del bilancio sociale: la logica degli organi di governo; le tecniche di coinvolgimento degli stakeholder; le modalità di rappresentazione dell'attività svolta; la struttura di rendicontazione formulata; le dimensioni di risultato analizzate.

Secondo la letteratura citata, il bilancio sociale dovrebbe essere uno strumento anche di governance interna, voluto dal vertice dell'ateneo e successivamente realizzato e condiviso da tutti i livelli dell'università. In tal senso ha operato l'Università di Bologna, che ha costituito un gruppo di lavoro nel quale sono confluite le diverse anime dell'ateneo (organi di governo, personale docente, personale tecnico-amministrativo, a vario livello distribuito nella scala gerarchica e tra i diversi settori di attività, studenti e laureati dell'Alma Mater), con competenze differenziate (controllo di gestione, rendicontazione sociale, settore pubblico, università e molte altre).

La definizione generale della struttura e del contenuto del documento si è rifatta ai principali modelli di rendicontazione sociale esistenti. In tal senso, l'Università di Bologna ha rendicontato gli indicatori richiesti dal G3.0. quali i dati sulla composizione e formazione del personale, i dati sulla produzione dei rifiuti e sui consumi, ecc.

L'aspetto più difficile da misurare è il "prodotto" delle università in termini di outcome: apprendimento e conoscenza che hanno rilevanti impatti sul benessere e sul progresso della società e sullo stato del territorio. Il caso di studio presentato in questo articolo ha delineato le modalità adottate dall'ateneo per tentare di rappresentare alcuni di questi impatti. 
In definitiva, il bilancio sociale dell'Università di Bologna è strumento per operare in maniera trasparente con gli interlocutori esterni (specie perché riesce a coinvolgere tutti i portatori di interesse), per rendicontare aspetti economici, sociali e ambientali, nonché mezzo utile per la governance interna.

I contributi dei lavori di questo numero di Management Control espongono i progressi e le criticità nella gestione e nel controllo delle università statali italiane. Le performance degli atenei sono analizzate: per come dovrebbero essere secondo una recente normativa, ma anche per come dovrebbero essere secondo principi di management and accounting non recepiti dal legislatore, che potrebbero supportare i processi decisionali interni (a livello di singolo ateneo) ed esterni (a livello ministeriale, anche in termini di politiche pubbliche).

Tra le altre criticità emerse: la difficile integrazione dei sistemi informativi utili per il controllo e per le decisioni. L'abitudine a rilevare e rendicontare le attività amministrative in COFIN, con assenza di valorizzazione degli aspetti economici e patrimoniali, è dura da superare come mentalità e processi organizzativi. La consuetudine di rilevare/valutare poco (oggi tanto) i processi della didattica e i processi della ricerca ha determinato una loro eccessiva autonomia, mentre dovrebbero essere integrati tra loro e con il più generale funzionamento degli atenei. I sistemi informativi animati ed alimentati da una visione integrata delle operazioni e dei processi aziendali sembra essere il futuro compito da svolgere anche in seno agli atenei, tutti gli studiosi di economia aziendale hanno il dovere di adoperarsi perché ciò avvenga.

\section{Bibliografia}

Agasisti T., Catalano G. (2013), Debate: Innovation in the Italian higher education system: introducing accrual accounting, Public Money \& Management, 33, 2, pp. 92-94. Doi: 10.1080/09540962.2013.763414.

Cantele S., Martini M., Campedelli B. (2012), Gli atenei italiani e gli strumenti di pianificazione e controllo: a che punto siamo?, Management Control, 2, 1, pp. 55-83.

Doi: 10.3280/MACO2012-001004.

De Bruijn H. (2002), Performance Measurement in the Public Sector: Strategies to Cope with the Risks of Performance Measurement, International Journal of Public Sector Management, 15, 7, pp. 578-594. Doi: 10.1108/09513550210448607.

Del Sordo C., Siboni B. (2013), La rendicontazione sociale nel sistema universitario italiano. Analisi critica degli studi e delle esperienze, in Cassone A., Sacconi L., a cura di, 
Autonomia e responsabilità sociale dell'Università. Governance e accountability, Milano, Giuffrè.

Lawson R., Stratton W., Hatch T. (2003), The benefits of a scorecard system, CMA Management, 77, 4, pp. 24-26.

Marchi L., Greco G. (2012), Controlling and reporting for strategy, Management Control, 2, 3, pp. 5-6. Doi: 10.3280/MACO2013-SU3001.

Mussari R., Sostero U. (2014), Il processo di cambiamento del sistema contabile nelle università, aspettative, difficoltà e contraddizioni, Azienda Pubblica, 27, 2, pp. 125-147.

Mussari R., D’Alessio L., Sostero U. (2015), Il nuovo sistema contabile delle università, Azienda Pubblica, 28, 3, pp. 227-246.

Paolini A. (1993), Il controllo strategico. Uno schema di analisi, Milano, Giuffré.

Paolini A., Soverchia M. (2013), Le università statali italiane verso la contabilità economico-patrimoniale ed il controllo di gestione, Management Control, 3, 3, pp. 77-98. Doi: 10.3280/MACO2013-003005.

Trequattrini R., Nappo F., Lardo A. (2015), L'introduzione della contabilità-economico patrimoniale nelle università tra adeguamento alle norme e nuovi paradigmi emergenti: il caso Uniclam, Azienda pubblica, 28, 3, pp. 269-285. 